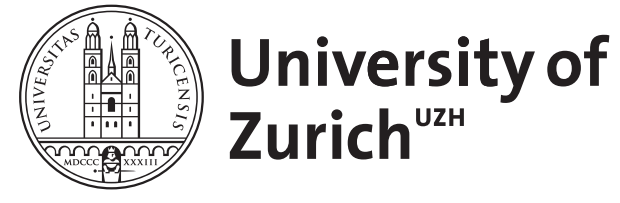

Zurich Open Repository and Archive

University of Zurich

University Library

Strickhofstrasse 39

CH-8057 Zurich

www.zora.uzh.ch

Year: 2014

\title{
Bilder-Denken und Symbolisierungsprozesse in der frühen
}

Kulturwissenschaft

\author{
Naumann, Barbara
}

Posted at the Zurich Open Repository and Archive, University of Zurich

ZORA URL: https://doi.org/10.5167/uzh-100708

Book Section

Originally published at:

Naumann, Barbara (2014). Bilder-Denken und Symbolisierungsprozesse in der frühen Kulturwissenschaft. In: Benthien, Claudia; Weingart, Brigitte. Handbuch Literatur Visuelle Kultur. Berlin: De Gruyter, 86-103. 


\title{
2.4 Bilder-Denken und Symbolisierungs- prozesse in der frühen Kulturwissenschaft
}

\author{
Barbara Naumann
}

\section{Einführung}

Aby Warburgs Bestimmung des Bildes als „Prägewerk seelischer Ausdruckswerte“ (Warburg 2012b [1926], 101), Walter Benjamins Deutung des Bildes als Instanz, in der „das Gewesene mit dem Jetzt blitzhaft zu einer Konstellation zusammentritt“ (Benjamin 1982 [1928-1940], 576) und schließlich Ernst Cassirers Charakterisierung des Bildes als einen „,bestimmten geistigen Brennpunkt“ im „fast unmerklichen Übergang zur Darstellung“ (Cassirer 1982 [1929], 126-127) markieren bedeutende Stationen einer Umorientierung im Umgang mit Bildern und Bildtheorien in der ersten Hälfte des 20. Jahrhunderts. Sie entstanden in zeitlicher Nähe zueinander und - im Falle Warburgs und Cassirers - im gemeinsamen Kontext der Renaissance- und Mythosforschung an der Kulturwissenschaftlichen Bibliothek in Hamburg.

Warburg stellt den seelisch-körperlichen, gestischen Ausdruck ins Zentrum des „Bilder-Denkens“ (Naumann und Pankow, 2004), Benjamin liest dem Bild die entscheidende Aussagekraft über historische Zusammenhänge und gesellschaftliche Konflikte ab, Cassirer schätzt das Bild als symbolische Form und hebt die bildliche Abstraktionsleistung als historisch-systematische Etappe auf dem Weg zur sprachlich-begrifflichen Darstellung hervor. Bei aller unterschiedlichen Akzentuierung wird deutlich, dass Bilder im Denken der drei Autoren eine zentrale Rolle einnehmen. Das Bild figuriert bei ihnen nicht mehr nur als Gegenstand kunsthistorischer, formalästhetischer und stilkritischer Analysen oder als Ausdruck des Paragone der Künste (vgl. Walzel 1917 sowie 3.1 WeTzEL). Schon lange bevor W. J. T. Mitchell mit der berühmt gewordenen Wendung vom pictorial turn (Mitchell 1992) der Tatsache Rechnung zu tragen suchte, dass das Denken über Bilder und mit Bildern gegenüber dem begrifflichen Denken keine defizitäre Form darstelle, sondern ein integraler Bestandteil der Erkenntniskritik und Kulturtheorie sein müsse, vollziehen die genannten Autoren in den 1920er Jahren eine entscheidende epistemische Aufwertung des Bildkonzepts (vgl. auch 3.2 GIL).

Bemerkenswert ist, dass Bilder beziehungsweise die ikonologische Analyse bei keinem der drei Theoretiker ein isoliertes Telos der Untersuchungen darstellt. Häufig entsteht die Auseinandersetzung mit dem Bild im engen Dialog mit literarischen Texten. Bilder fungieren als genuine Reflexionsmedien für sprachtheore- 
tische, mediologische und vor allem kulturwissenschaftliche Erkenntnisinteressen. Warburg, Cassirer und Benjamin stimmen darin überein, dass der Bildbegriff an zentraler Stelle in den Kontext der Ausdrucks- und Darstellungstheorie zu situieren sei. Die Reflexion der Krise des sprachlichen Ausdrucks führt sie darüber hinaus auf unterschiedlichen Wegen zur Neubewertung der epistemischen Rolle der Bilder. In Verbindung damit steht der Dialog mit bedeutenden Werken der Literatur: Insbesondere Benjamin entwickelt in seinen Studien zu Charles Baudelaire, Marcel Proust und Franz Kafka eine Reihe von erkenntniskritisch einschlägigen Bildbegriffen.

Die Vieldimensionalität des Bilder-Denkens ergibt sich insbesondere aus dem Umstand, dass das Phänomen der Bildlichkeit in der ersten Hälfte des 20. Jahrhunderts in ganz unterschiedlichen akademischen Disziplinen eine rege Aufmerksamkeit hervorrief. Warburg, Cassirer und Benjamin waren mit ihrer interdisziplinären und kulturwissenschaftlichen Orientierung für diese Entwicklung paradigmatisch: zugleich unkonventionell, innovativ und zukunftsweisend. Ihre kulturwissenschaftliche Bildwissenschaft, die sich im Dialog mit der Literatur entwickelte und im Falle Benjamins mit dem Buch Berliner Kindheit um Neunzehnhundert (1932-1938) auch zu einer neuen Literatur führte, stellt für die Medien- und Kulturdebatten bis heute eine Herausforderung dar.

\section{Kunst und Affektbewältigung, Medialität und Mnemosyne: Aby Warburg}

Warburgs Bildwissenschaft aktualisiert das mehrdeutige Konzept des Bilder-Denkens in einer ganz eigenständigen Weise: Zum einen stellt er die wissenschaftliche Untersuchung von Bildern, Skulpturen, Riten und anderen Ausdrucksgebärden ins Zentrum der Aufmerksamkeit. Zum anderen entwickelt Warburg neue Möglichkeiten einer sich bildhaft darstellenden Wissenschaft. Auf eine solche Verbindung von Bildlichkeit und Wissen angelegt waren Warburgs Ausstellungsprojekte, die „Bildersammlung zur Geschichte von Sternenglaube und Sternenkunde“, der unvollendete „Mnemosyne-Atlas“ und nicht zuletzt die innovative Organisation und Farb-Systematik der von ihm gegründeten Kulturwissenschaftlichen Bibliothek Warburg“ (K. B. W.).

Ungewöhnlich zu seiner Zeit waren Warburgs umfangreiche kulturwissenschaftliche Forschungen, deren viele Facetten vielleicht am besten unter den Begriffen einer Psychohistorie der Kunst und einer Stilistik der Kultur gebündelt werden können. Grundlagen der kulturtheoretisch informierten Wendung der Bildwissenschaft bildeten Warburgs Studium bei dem Religionswissenschaftler 
Hermann Usener, dessen Überzeugung von einer psychologischen Motivation der antiken Mythenbildung er folgte, und Jacob Burckhardts „synthetische Kulturgeschichte“ (Warburg 1998 [1932], 193). Ebenfalls wegweisend wurden Friedrich Nietzsches und Sigmund Freuds Überlegungen zum Zusammenhang von Affektkontrolle und Kulturleistung. Warburg, der ausgebildete Kunsthistoriker und Renaissancespezialist, folgt von Beginn an Fragestellungen, die ihn an die Randzonen der etablierten akademischen Disziplinen führen. Er interessiert sich für den Ausdruck starker Emotionen in der Kunst und deren Überlieferung von der Antike bis zur Gegenwart (vgl. Warburg 1998 [1932]). Er verbindet damit die Wahrnehmung des kulturellen Kontexts der Werke sowie die Verabschiedung eines präskriptiven ästhetischen Ideals der Antike, etwa desjenigen Winckelmannscher Prägung.

Warburg sieht in der Kunst in erster Linie ein Überlieferungsmedium konfligierender Emotionen und Situationen. Paradigmatisch findet er dies in der florentinischen Renaissancekunst dargestellt: Die Renaissance als eine Zeit des Umbruchs, des Konflikts zwischen mittelalterlicher christlicher Glaubensbefangenheit, heidnischen Mysterien, antiker Kosmologie und neuplatonischer Philosophie war für ihn eine Schlüsselepoche. An ihrer widerspruchs- und bildfreudigen Kultur erkennt er eine überindividuelle kulturelle Dynamik: einen Prozess des energetischen Austauschs, der den Kontakt mit stark geprägten Formen, also der Kunst, begleitet. Warburgs Begriff für solche affektiv aufgeladenen Formen ist die „Pathosformel“ (vgl. Warburg 1992 [1912], u. a. 125-126, 153, 173). In vielfachen terminologischen Varianten durchzieht die darin gebündelte Frage nach der Bewältigung von Angst sein gesamtes Werk. Warburgs Forschung gilt den „Prägewerken seelischer Ausdruckswerte“(Warburg 2012b [1926], 101), zu denen er nicht nur Kunstwerke zählt, sondern auch kulturell sedimentierte Formen wie Rituale und Tänze. Das übergreifende Ziel seiner Unternehmungen ist eine „energetische Lehre menschlichen Ausdrucks“, und zwar auf der „Grundlage einer philologisch-historischen Untersuchung des Zusammenhangs zwischen der bildhaftkunstwerklichen Gestaltung und der Dynamik des wirklichen oder dramatisch gestaltenden Lebens“ (Warburg 2012b [1926], 101). Eine „rein formale Ästhetik“ (ebd.), wie sie in der Kunstgeschichte seiner Zeit dominant war, klassifiziert er als „steriles Wortgeschäft“, angesiedelt zwischen „Religion und Kunstausübung“ (Warburg 1988 [1923], 65); für die damit in Verbindung stehende „ästhetisierende Kunstgeschichte“ empfindet er ,aufrichtigen Ekel“ (ebd.).

Das kritische Verhältnis zur Kunstgeschichte fand seinen Niederschlag in den Notizen zu einem Vortrag über das Schlangenritual der Hopi-Indianer. Darin kristallisiert sich Warburgs Interesse, die formalästhetisch orientierte Bildwissenschaft durch kulturwissenschaftliche Breite und psychologische Sensibilität zu erweitern. Die „Ausweitung des Gegenstandsbereichs auf nicht-künstlerische 
Bilder“, auf „Lebensstile, Rituale, habituelle Muster“ (Hensel 2011, 13) und auf „Bild- und Wortquellen aller qualitativen Grade und medialen Formen“ (Böhme 1997, 140) - mithin ein ebenso interkulturelles wie intermediales Interesse -, profilieren von Beginn an seine Forschungen. Seine Theorie sucht die „Gesamtkultur“ (Warburg 1992 [1912], 406) zu erschließen und stellt als deren allgemeinste und stärkste affektive Bewegung die Angstbewältigung ins Zentrum. Hierin schließt Warburg an die großen Theorien des 19. und frühen 20. Jahrhunderts an, die die kulturelle Formierung im Zusammenhang mit dem sich selbst problematisierenden Subjekt entwerfen und die vor allem mit Namen wie Søren Kierkegaard, Nietzsche und Freud verbunden sind.

Ähnlich wie Cassirer sieht Warburg im ,Ausdruck‘ ein Konstrukt der Affektbearbeitung, das als komplexe symbolische Form zu verstehen ist. Doch gibt es erhebliche Unterschiede zwischen beiden Denkern. Cassirer versteht den Prozess der Symbolisierung als eine anthropologisch verankerte Möglichkeit, von der Irrationalität der Affekte frei zu werden; optimistisch setzt er auf eine Versöhnung von vernünftigen und irrationalen Strebungen im konstruktiven Akt der Schaffung symbolischer Formen. Warburg hingegen betont im Symbol die Latenz einer immerwährenden Bedrohung. Sie dauert selbst in jenen Refugien an, die dem unmittelbaren Zwang, der Angst und dem Mythos abgerungen wurden. Wie die italienische Renaissance, so sah Warburg auch das Denken der Moderne mit der „Erbmasse phobischer Engramme“ konfrontiert (Warburg 2012a [ca. 1927], 4).

Den Pathosformeln kommt die Funktion einer ambivalenten Bewältigungsstrategie zu: Sie sind Teil des Verhaftetseins im affektiven Zustand, machen diesen aber auch sichtbar und finden eine Form für den Konflikt, die Angst, die Phobien, das starke Potential der Selbstzerstörung oder das Gefühl der Schuld. Georges Didi-Huberman hat vorgeschlagen, das Symbolische der Pathosformeln als Verkörperung des Symptomalen zu verstehen, als das „konkrete Symptom einer Spaltung, die in der ,Tragödie der Kultur‘ permanent geschieht“ (DidiHuberman 2004, 209). Die Überlieferungsgeschichte der Pathosformeln, das Nachleben der Antike - sei es in den Kunstwerken der Renaissance, sei es in den Ritualtänzen der Hopi -, wäre dann vor allem eine Geschichte der Symptome, die sich unter dem Regime der Dialektik von Verdrängung und Wiederkehr des Verdrängten vollzieht und die Kunst in diesem Prozess als eine ,,plastische Kompromißformel[]،“ (ebd., 211) in Erscheinung treten lässt.

Pathosformeln durchziehen nach Warburg die großen historischen und kulturellen Räume von der Antike über die Renaissancekunst Italiens bis zu den Riten, Artefakten und alltagskulturellen Objekten der Gegenwart. Die bildliche Ausdrucksdarstellung interessiert ihn nicht aufgrund ihrer ästhetischen Formalisierung, sondern aufgrund der Tatsache, dass in ihr emotionale wie gestische ,Bewegung' festgehalten ist. Schon die Dissertation zu Sandro Botticelli von 
1893 akzentuierte eine Theorie des bedeutsamen „bewegten Beiwerks“ - etwa flatternde Haare und Gewänder (vgl. Warburg 1998 [1932], u.a. 54; siehe dazu auch Didi-Huberman 2010 [2002]). Warburg versteht die Pathosformel nicht nur als Körperbild oder Gebärdensprache. Er sieht in ihr die Überschreitung „hin zu einem umfassenderen Begriff des Stils als ,Ausdrucksweise““ (Zumbusch 2004, 184) und von der Affektbannung der Kunst erwartete er nicht weniger als die Eröffnung eines „Denkraums“(ebd., 59).

Die Auseinandersetzung mit der Tradierung von Pathosformeln hat Warburg mit einem zweiten umfangreichen Forschungsinteresse verbunden: der Geschichte der Magie, der Astrologie und dem antiken Sternenglauben. Damit in Zusammenhang steht die Innovation von kulturwissenschaftlichen Darstellungstechniken, die die zeitlich-räumliche Dimensionen der Überlieferung im Zuge eines cultural mapping nachzeichnen. Als ein bedeutendes Beispiel sei die Studie „Italienische Kunst und internationale Astrologie im Palazzo Schifanoja zu Ferrara“ genannt (Warburg 1992 [1912], 173-198), die den Einbruch der astrologischen Bildwelten Ägyptens und des Vorderen Orients in die christliche Ikonografie synchron - als Bildschichtung - und zugleich diachron - als 1500 Jahre dauernde Wanderbewegungen der Bilder - beschreibt. Warburg selbst charakterisierte seine ,kulturwissenschaftliche Methode‘, ähnlich wie Freud, gern mit Rückgriff auf das Vokabular der Archäologie. Deren Erkenntnisinteresse führt „durch die Schichten der historischen Lagerung (zeitlich-räumlich als Querschnittseinheiten) zurück auf den Urgrund denkraumzerstörender magischer Praktik“ (unveröff. Manuskript Warburgs, zit. bei Zumbusch 2004, 50), die den menschlichen gebärdensprachlichen Ausdruck durch realen Handlungsreiz hervorbringt.

Die Bildersammlungen des Mnemosyne-Atlas, die Warburg über Jahrzehnte seines Forscherlebens zusammengestellt hat, suchen die religiösen und artistischen Spuren nachzuzeichnen, in denen die „Dauerfunktionen“ (Warburg 2012a [ca. 1927], 3) des menschlichen Leidens ihren formelhaft-pathetischen Ausdruck finden (Abb.1). Hierin zeigt sich ebenso ein kulturhistorisches wie mediologisches Engagement, denn der Atlas ist für Warburg das Medium par excellence, um den Thesen zum Nachleben der Antike Evidenz zu verleihen und sein bildwissenschaftliches Denken zu demonstrieren. In der Ausstellung des Mnemosyne-Atlas vermochte die Betrachterin oder der Betrachter zunächst einmal nichts anderes als thematisch organisierte Serien von Abbildern (Fotografien, Reproduktionen) unterschiedlicher Provenienz und Qualität zu erblicken. Man kann den Bilderatlas deshalb auch als „Schatz- und Wunderkammer des kulturellen Gedächtnisses“ verstehen (Böhme 1997, 153), eine Kammer zudem, die ihre Materialien nach assoziativen und Kontiguitäts-Gesichtspunkten ordnet und ästhetisch enthierarchisiert. In jedem Fall zeigt sich in Warburgs assoziativem 
Verfahren der suchende, vagierende Charakter einer in Entwicklung befindlichen Bildwissenschaft. Warburg betitelte die Bilderserie: „Mnemosyne, Bilderreihe zur Untersuchung der Funktion vorgeprägter antiker Ausdruckswerte bei der Darstellung bewegten Lebens in der Kunst der europäischen Renaissance“ (Warburg 2012a [ca. 1927], XVIII). Wie die Überfülle der Titelbegriffe zeigt, meint ,Bild‘ in diesem Zusammenhang unter anderem: Prägung, Engramm der starken Gefühlsbewegung und zugleich - darin auf Mnemosyne bezogen - deren Wiedererinnerung und lebendige beziehungsweise bewegte ,Re-Aktualisierung، Die gestisch-bildliche Überlieferung der Pathosformeln von der Antike bis zur Moderne sind ebenso in den Atlas eingegangen wie - aufgezeichnet in Landkarten - deren räumliche, geografische und topologische Überlieferungswege. Mit dem neuartigen Arrangement der Bilder soll der „Austausch der Ausdruckswerte zwischen Norden und Süden“ als ein „vitaler Vorgang im Kreislaufprozess der europäischen Stilbildung“ verdeutlicht werden (Warburg 2012a [ca. 1927], 5). Warburg zufolge ist dieser motiviert durch die Dynamik einer tragischen, bipolar ausgespannten Kulturentwicklung, die zwischen dem „Ausdruckszwang“ und dem „Ordnungsbedürfnis“ der Sophrosyne oszilliert (Böhme 1997, 153). Ähnlich wie Nietzsche und Jacob Burckhardt situiert auch Warburg die Triebkräfte der Kultur in der Leidensbewältigung und deren symbolischer Formierung, ihrer Erinnerung und immer neu durchlebter Aktualisierung. Die historische Veränderung der Formensprachen im Zuge der Überlieferung, das Formieren der Kultur als Stil, wird in diesem Modell ebenso adressiert wie die Integration des mythischen Denkens in das Nachleben der Antike. So ist es bereits bei Nietzsche zu lesen. Über Nietzsche hinaus aber offeriert Warburg mit dem Mnemosyne-Atlas einen neuen, bild-, kultur- und mediengeschichtlich bedeutsamen Wissensspeicher. Die Geschichte des durch Affekte geformten Ausdrucks ist darin nicht nur beschrieben. Sie ist durch die Komposition der Bilder zugleich erinnert, dargestellt und zur Anschauung gebracht.

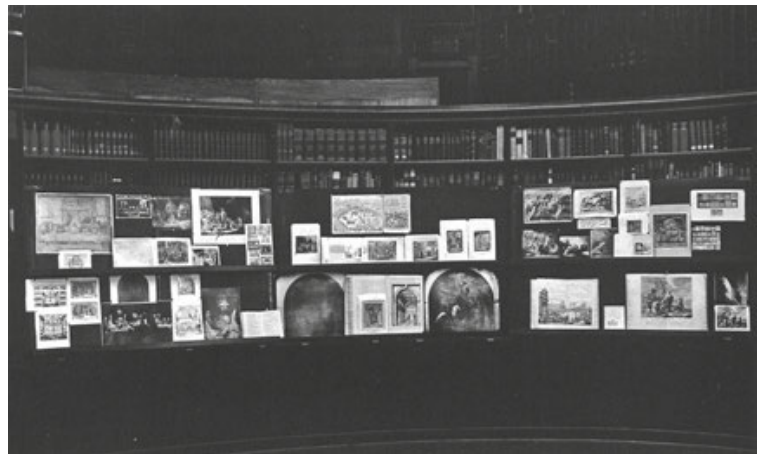

Abb. 1: Aby M. Warburg: Mnemosyne-Atlas, 19241929, Tafeln der RembrandtAusstellung, 1926 


\section{Symbolische Form, symbolische Prägnanz - kunstphilosophische Grundlagen: Ernst Cassirer}

Zwischen Warburgs ikonologischer Bildwissenschaft und dem symboltheoretischen Denken Cassirers gibt es zahlreiche Bezüge. Cassirer begann die Zusammenarbeit mit der K. B. W. während der Arbeit am zweiten Teil der Philosophie der symbolischen Formen, Das mythische Denken (1924), und fand in der Sammlung „nicht nur ein reiches, in seiner Fülle und Eigenart fast unvergleichliches Material“ vor; überdies erschien ihm dieses Material in seiner „Gliederung und Sichtung, in der geistigen Prägung, die es durch Warburg erhalten hat, auf ein einheitliches geistiges Problem bezogen, das sich mit dem Grundproblem [s]einer eigenen Arbeit aufs nächste berührte“ (Cassirer, 1987 [1925], XIII). Im Unterschied zu Warburg entwickelte Cassirer keine explizite Theorie des Bildes oder der bildenden Kunst. Vielmehr integrierte er sämtliche bildtheoretische Überlegungen in die Konzeption eines symbolischen Prozesses, der zugleich als Erkenntnisund als Konstruktionsprozess der Kultur zu denken sei.

Cassirers Symboltheorie antwortet auf die Grundsatzfrage nach der Genese von Bedeutung. Eine Antwort, die Gültigkeit über alle Ausdifferenzierung der einzelnen symbolischen Formen hinweg beansprucht, liefert der Begriff der „symbolischen Prägnanz“. Darunter versteht er „die Art [...], in der ein Wahrnehmungserlebnis, als ,sinnliches' Erlebnis, zugleich einen bestimmten nichtanschaulichen ,Sinn' in sich faßt und ihn zur unmittelbaren konkreten Darstellung bringt“ (Cassirer 1982 [1929], 235). Wahrnehmung bestehe nicht in „bloß ,perzeptive[n]' Gegebenheiten, denen später irgendwelche ,apperzeptiven' Akte aufgepfropft wären und durch die sie gedeutet, beurteilt und umgebildet würden“ (ebd., 235). In der Philosophie der symbolischen Formen gibt Cassirer unterschiedliche, nämlich anthropologische, zeichentheoretische, erkenntnistheoretische und phänomenologische Antworten auf die Frage nach dem Konnex von Sinnlichkeit und Sinn. Allen aber ist gemeinsam: Sie verwerfen die Annahme eines transzendentalen Signifikanten und akzeptieren Bedeutung allein als Element des Symbolprozesses. In jeder sinnhaften symbolischen Form - der Sprache, dem Mythos, der Kunst, der Wissenschaft - verbindet sich die prägende Spur der materiell-sinnlichen Seite mit einem abstrakten, nicht-anschaulichen ,Sinn'.

Den Künsten misst Cassirer aufgrund ihres symbolischen Charakters epistemische Valenz zu. Demnach liege es in der Logik des Symbolprozesses, dass die Kunst über die Nachahmung hinausgehe. Ihre gestalterische Lösung von der Unmittelbarkeit der ersten Eindrücke impliziere einen Bruch mit dem traditionellen Mimesis-Gebot. Cassirer situierte die „Wechselbestimmung“ (Cassirer 1983[1921-1922], 235) zwischen Bezugsrahmen und einzelnem Phänomen innerhalb dieses Rahmens als Grundlage jeden Symbolisierungsprozesses und 
jeder Semiose. In direkter Anlehnung an Johann Wolfgang von Goethes Symbolauffassung und Darstellungstheorie - wie sie unter anderem in dessen kleinem wissenschaftstheoretischen Versuch als Vermittler zwischen Subjekt und Objekt (1792) niedergelegt ist - erläutert Cassirer, dass die „phänomenale[] Beschaffenheit“ eines Datums immer „schon von der Ordnung abhängig“ sei, in der sie steht. Die „reine Erscheinungsweise“ der Farbe zum Beispiel werde „durch eben diese Ordnung [der Farben untereinander, d. V.] bestimmt“ (Cassirer 1982 [1929], 234-235).

Die Ausführungen zur allgemeinen Funktion der symbolischen Prägnanz bieten den bildwissenschaftlich bedeutsamsten Aspekt der Symbolphilosophie. Unübersehbar ist die Verwandtschaft des phänomenologisch konturierten Begriffs der symbolischen Prägnanz mit Warburgs Konzept der Pathosformel. Warburg beschrieb die Formwerdung des Pathos als einen Transformationsprozess des gestischen Ausdrucks. Für Cassirer wie Warburg geht es darum, den symbolischen Prozess als Dynamik des kulturellen Wandels zur Geltung zu bringen. Cassirer zieht aber aus der Beschreibung der Formgenese andere kulturtheoretische Schlüsse. Unübersehbar ist die Differenz zu Warburgs Denken vor allem im Hinblick auf die Funktion der Angst und der Affektkontrolle. Cassirer sieht die Freiheit des Menschen als durch die Aufklärung rationalisierte anthropologische Gegebenheit; er erklärt sie zur Grundlage des animal symbolicum. Warburg hingegen leitet die Energien zu Formgebung und Wandel aus dem Zwang zur Affektkontrolle und hier vor allem aus dem Aspekt der Angstbewältigung her.

Cassirer geht davon aus, dass sich die symbolischen Formen in einem „dreifachen Stufengang“ entfalten; die Formen Mythos, Sprache und Kunst durchlaufen diesen Stufengang als einen semiotischen Prozess, der je spezifische Relationen zwischen „Zeichen und Bezeichnetem“ ausprägt (Cassirer 1983 [1921-1922], 178). Von der ersten Stufe, auf der sich das Zeichen dem Bezeichneten „möglichst nahe anschmiegt“, um es ,gleichsam in sich aufzunehmen“ (ebd.), führt die Entwicklung über eine zweite, „analogische“ Stufe, die schon eine „Subjektivität des Denkens“ erkennen lässt, aber immer noch nach einer „Entsprechung“ zum Bezeichneten sucht (ebd., 180). Auf der dritten, abstrakten Stufe schließlich wird ,auf jede Form der wirklichen Nachahmung [...] verzichtet, und statt dessen tritt die Funktion der Bedeutung in reiner Selbständigkeit hervor“ (ebd., 182). Cassirer stellt diese allgemeine semiotische Stufung auf dem Gebiet der Sprachentwicklung dar und erkennt in der Sprachgeschichte die Dynamik einer zunehmenden Abstraktionsleistung, die sich über die Schritte der mimetischen, analogischen und rein symbolischen Form - auch Darstellungsform genannt - vollzieht (vgl. Cassirer 1988 [1923], 5, 1982 [1929], u. a. 126-127).

Cassirers Insistenz auf die Zusammengehörigkeit von Theorie und Anschaulichkeit im symbolischen Prozess, die interdisziplinäre, Natur- und Geisteswis- 
senschaften einbeziehende Ausrichtung seiner Philosophie, und schließlich der poietische Kern des Symbolisierungsvorgangs, der sich im Konstruktionscharakter seiner Philosophie als Kulturtheorie zeigt, sind Bausteine einer allgemeinen Theorie des Bildes. Entscheidende Aspekte dieser Gedankenkette verdanken sich zudem einem konkreten literarischen Werkkomplex, nämlich der lebenslangen Auseinandersetzung Cassirers mit den Werken Goethes. Diese Auseinandersetzung zieht sich als ein roter Faden durch Cassirers Schriften und bezieht sowohl die Dichtung als auch die naturwissenschaftlichen Schriften Goethes ein. Goethes Werk verkörpert nach Cassirer das, was die Symbolphilosophie im Einzelnen zu zeigen versucht (vgl. Naumann 1998, 98; siehe auch Cassirer 2003a [1922], 2003b [1940-1941], 2006 [1925-1944; bes. 1934 und 1935]). Nicht selten nutzte er Texte und Theoreme Goethes als Sprachmasken für eigene Argumentationen und sah in ihnen die Paradigmen einer modernen Kulturphilosophie vorgeprägt. Eine Affinität zu seinen eigenen theoretischen Interessen erkannte er nicht zuletzt in Aspekten, die für eine bildtheoretische Diskussion relevant sind, wie Goethes expliziter Auseinandersetzung mit dem Symbolbegriff, der Entfaltung des Denkens in Stufen zunehmender Abstraktion - zum Beispiel in Goethes Aufsatz Einfache Nachahmung der Natur, Manier, Stil (1789) -, weiterhin im Vorentwurf eines interdisziplinären Dialogs zwischen Natur- und Geisteswissenschaften, und schließlich in der kritischen Diskussion von Kants Transzendentalphilosophie. Cassirer bedient sich der Denk-Figuren Goethes, um zu zeigen, „was als symbolische Kulturphilosophie im umfassenden Sinne zu verstehen sei“ (Naumann 1998, 12).

In der Kunst sieht Cassirer zwei unterschiedliche Aspekte zusammenkommen: Sie sei ebenso unmittelbarer oder „intuitiver“ Ausdruck wie „Rationalität der Form“ und besitze in dieser Doppelung ihren spezifischen Erkenntnismodus (Cassirer 1990 [1944], 260 und 257). Die bildende Kunst vermöge „die Dinge zu visualisieren, statt sie nur zu konzeptualisieren“ (ebd., 261). Wie der Symbolisierungsprozess generell bringt auch die Kunst im Speziellen zugleich eine performative und mediale Seite zur Erscheinung. Cassirer begreift Ausdruck zugleich als Form; jeder Ausdruck besitze eine Gestalt, einen „bestimmten ,physiognomischen“ Charakter“ (Cassirer 1982 [1929], 526). Der wesentliche Unterschied zu Warburgs Bildtheorie liegt in der Annahme, dass die Leidenschaft „ohne Zwang“ (Cassirer 1990 [1944], 229) in eine formenbildende Kraft verwandelt werden könne. Die freie Bindung aller Ausdrucksgesten an die Form und schließlich an den artistischen Werkcharakter machte für ihn das Kunstwerk aus.

Bemerkenswert ist Cassirers ebenfalls an Goethe angelehnte Wertschätzung des lebendigen Charakters der Kunst, die für ihn, ähnlich wie für Warburg, eine Ausdrucksform des bewegten Menschen ist (vgl. Recki 2004, u. a. 115). Er sieht die Kunst als Zeugnis der menschlichen „Empfänglichkeit für das dynamische 
Leben von Formen“ (Cassirer 1990 [1944] 232); wie Warburg interessiert ihn die „Lehre vom bewegten Menschen“ (Krois 2011, 78). Keine Rolle spielt hingegen für Cassirers Kunst- und Bildkonzept die Ästhetik der Mortifikation, der Stillstellung, die seit Gotthold Ephraim Lessings Schrift Laokoon oder Über die Grenzen der Malerei und Poesie (1766) den Vergleich der bildenden Kunst mit der Dichtung maßgeblich bestimmt hatte (siehe dazu 2.3 SchneIDER). Ganz auf Lebendigkeit, Dynamik und Formwandel orientiert, hat für Cassirer das Bild, hat überhaupt die bildende Kunst nicht das letzte Wort in Bezug auf die Erkenntnis- und Deutungsfunktion: „Eine künstlerische Form [...] entsteht erst dort, wo die Anschauung sich von jeder Gebundenheit im bloßen Eindruck gelöst, wo sie sich zum reinen Ausdruck befreit hat.“ (Cassirer 1983 [1921-1922], 182) Der ,reine Ausdruck‘, die ,reine Bedeutung“ wird von Cassirer in der „Philosophie der symbolischen Formen“ gleichgesetzt mit der Darstellungsfunktion der Sprache.

In der Architektur der symbolischen Formen besetzen also die symbolische Prägnanz - und damit ein Begriff, der bildliche Aspekte in sich aufgehoben hat -, und die Sprache in ihrer abstraktesten Funktion, der Darstellungsfunktion, die entscheidenden Positionen. „Denn solange die Repräsentation, die Darstellung als solche, noch eines bestimmten Anschauungsbildes ihres Trägers bedarf, hebt sie sich nicht scharf und prinzipiell von diesem ihrem Substrat ab. Der Blick des Geistes verfängt sich allzu leicht in die Einzelheiten des Bildes selbst. Erst die Sprache bringt hierin eine neue und entscheidende Wendung“, so Cassirer, denn sie sei „sozusagen mit keiner eigenen sinnlichen Materie mehr belastet“ (Cassirer 1982 [1929], 385). Cassirer möchte die Tendenz zur zunehmenden Abstraktion im Erkenntnisprozess zum Ausdruck bringen. Mit der Sprache verbindet er - ,sozusagen' - den Horizont einer bild- und materielosen Erkenntnis; doch kehrt er regelmäßig zur Notwendigkeit der bild- und materialhaltigen symbolischen Prägnanz zurück.

Gleichwohl zeigt sich in aller Deutlichkeit, dass Cassirers Beitrag zur Bildwissenschaft eingefügt ist in die allgemeine Differenzierung und Entwicklung symbolischer Formen, die er als übergreifendes kulturelles Prinzip verstand. Das Bild wird aus systematischen Gründen, nämlich im Hinblick auf seine Abstraktionsfähigkeit, der zu größerer Abstraktion fähigen sprachlichen Darstellungsfunktion nachgeordnet. Cassirer sieht das Bild lediglich als ein Element innerhalb der Pluralität möglicher symbolischer Formen. Die Philosophie der symbolischen Formen vollzieht keinen iconic turn. So sehr aus heutiger Sicht eine explizite mediale Reflexion des Bildbegriffs als Baustein einer Theorie der visuellen Kultur vielleicht wünschbar wäre - man kann Cassirer dessen Fehlen nicht zum Vorwurf machen, denn der Begriff der symbolischen Prägnanz nimmt sowohl mediale als auch erkenntniskritische Perspektiven auf. 
Mit den zum Teil ähnlich orientierten Studien Warburgs verbindet Cassirers Bildtheorie, dass beide die von ihnen besonders akzentuierte Lebendigkeit der Kunst in Zusammenhang bringen mit einem kulturell informierten Begriff des Stils. Geschult an Goethe wollte Cassirer den „Begriff des Stils mit dem der Erkenntnis“ und des „geistigen Ausdrucks“ verknüpft sehen (Cassirer 1983 [19211922], 183). Den Begriff des Stils bezieht Cassirer auf ,jede Art des Fortschritts vom natürlichen Dasein zum geistigen Ausdruck“ (ebd., 185); er markiert insbesondere deren bewegliche, lebendige Entwicklung. Stil ist für Cassirer wie für Warburg kein repetitives Formeninventar und mehr als ein bloß subjektives Ausdrucksgebaren. Der Stil erschließt eine in allen symbolischen Formen auffindbare symbolische Form.

\section{Bilder-Denken und Sprachkritik: Walter Benjamin}

Im Werk Benjamins findet sich eine Fülle bildtheoretischer Überlegungen. Neben der Extensität und Intensität des Bilder-Denkens ist es die Heterogenität der Bildkonzepte, die seine Schriften prägt. Seine philosophische und ästhetische Orientierung und entsprechend auch sein Bilder-Denken unterliegen einem deutlichen historischen Wandel von einer theologisch-sprachspekulativ orientierten Position zu einer an der materialistischen Ästhetik ausgerichteten Geschichtsphilosophie. Stets jedoch erwächst es aus dem direkten Zusammenhang mit seinen kritischen Schriften zur Literatur und Literaturtheorie, zur Geschichte und Philosophie. Benjamin verfolgt bildtheoretische Interessen nicht in erster Linie mit dem Ziel, eine kunsthistorisch oder ikonologisch informierte Analyse von BildKunstwerken zu leisten. Malerei als Thema zum Beispiel nimmt in Benjamins Schriften keinen breiten Raum ein. Jedoch finden zahlreiche Werke der bildenden Kunst in bedeutsamer Weise Eingang in seine Schriften (vgl. Weigel 2008, 265-296 und 297-332). Zu nennen wäre hier unter anderem die Aquarell-Zeichnung „Angelus novus“ (1920) des Paul Klee, die in Benjamins Besitz war und ihn zu geschichtskritischen Überlegungen anregte. Klees Angelus-Figur wurde für Benjamin zu einem „Vorstellungsbild“, das er mit historischen und theologischen Spekulationen verknüpfte (vgl. Benjamin 1980e [1925], 276). Als „Engel der Geschichte“ tritt er in den geschichtsphilosophisch orientierten Thesen „Über den Begriff der Geschichte“ (1942) auf. Mit dem Rücken zur Geschichte gewandt, sieht der ,Angelus novus“ keinen historischen Fortschritt, sondern erblickt nur „eine einzige Katastrophe“, die „unablässig Trümmer auf Trümmer häuft“. Er wird von einem Sturm fort vom Paradies und „unaufhaltsam in die Zukunft“ geweht (Benjamin 1980d [1942], 697). Benjamins Denkbild des Engels gewinnt 
gegenüber der pikturalen Darstellung Klees ein deutliches Eigenleben und beansprucht nicht, Bildinterpretation zu sein. Der ,Angelus novus', transformiert in ein erinnertes Vorstellungsbild, erhält neue Geltung als eine geschichtsphilosophische Denkfigur. Sie gemahnt an die Toten und an die Geschichte als fortlaufenden Zerstörungsprozess.

Einen ebenso wichtigen Punkt im Bilder-Denken Benjamins markiert Albrecht Dürers Kupferstich „Melencolia I“ (1514), dem in der Habilitationsschrift Ursprung des deutschen Trauerspiels (Benjamin 1980e [1925], bes. 329-335) die Rolle eines epistemisch bedeutsamen Denkbildes zukommt. Benjamin beschreibt die DingSymbole auf diesem für ihn ,unsagbar tiefen, ausdrucksvollen Blatt“ (Benjamin 1993, 143), um an ihnen die Geschichte des Deutungswandels in der Renaissance zu illustrieren. Dürers „Melencolia“ sei umgeben von „Bruchstücken der Dingwelt als ihren eigensten, sie nicht überfordernden Gegenständen“ (ebd., 334) und machten einen dialektischen Umschlagspunkt deutlich, an dem sich die „Allegoriefülle des Barock“ im Renaissancebild bereits einen Weg bahnt (ebd., 331). Der Stich vermag Benjamin zufolge einen historischen Mentalitätswandel deutlich zu machen: die Umdeutung der saturnischen entscheidungsunfähigen Melancholie hin zu einer Figur, die „um des Wissens willen“ die Welt verrät (ebd., 334).

Den Ausgang nahm Benjamins Auseinandersetzung mit Bildlichkeit bei der barocken Allegorie, dem Gegenstand des Trauerspielbuches. Im Zuge dieser Argumentation formuliert er eine Reihe kritischer Vorbehalte gegen den Symbolbegriff. Insbesondere das metaphysische beziehungsweise theologische Modell des Symbols, wie es durch die Romantik geprägt wurde, habe die artistische und ästhetische Deutung des Symbols in den Hintergrund gerückt. Benjamin verwirft den „Mißbrauch“ des Symbols als „Erscheinung einer Idee“ (ebd., 336). In einer Welt ohne Heilsversprechen könnten die Zeichen nicht auf Göttliches, das Symbol nicht auf Transzendenz verweisen, sondern nur noch auf sich selbst. Dieser Denkform werde die Allegorie, die die Erinnerung an die unerlöste Leidensgeschichte der Welt festhalte, gerechter (vgl. dazu auch 4.9 BEHRMANN).

Mit deutlichen Akzentverschiebungen setzt Benjamin die Allegoriediskussion im Essay über Goethes Wahlverwandtschaften (1924) und in späteren Schriften fort. In den Arbeiten zu Baudelaire und zur Analyse von Paris als Hauptstadt des 19. Jahrhunderts geht es ihm um das Geschick der Allegorie in der Moderne. Zwar teilt nach Benjamin Baudelaires Gebrauch der Allegorie mit der barocken Allegorie noch die Ablesbarkeit aus der Dingwelt, doch eigne ihr ein zerstörerischer Zug: Ihr Schauplatz seien die Heteronomien der Großstadt, sie gebe sich nur mehr im Fragment, im augenblickshaften Aufblitzen zu erkennen, sie trage Spuren des „Ingrimms“ und suche ihre Gegenstände, „ihre harmonischen Gebilde in Trümmer zu legen“ (Benjamin 1980f [1938], 671). Die moderne Allegorie Baudelaires „zerstört, ohne zu bewahren“ (Zumbusch 2004, 272). 
Die Kristallisation des Bildes als augenblickshaftes Innehalten im Fluss der Wahrnehmung mündet in das bedeutende Konzept des dialektischen Bildes. Im vielzitierten Satz aus dem Passagen-Werk - „Bild ist die Dialektik im Stillstand“ (Benjamin 1982 [1928-1940], 578) - wird das Bild nicht als materiale pikturale Darstellung (Zeichnung, Tafelbild etc.) in Anspruch genommen, sondern als Unterbrechung, als Diskontinuität: Es erscheint blitzhaft als „Konstellation“ aus „Gewesenem“ und „Jetzt“ (ebd., 576). Im Begriff des dialektischen Bildes kristallisiert sich das Interesse, die Geschichte - in diesem Fall die Geschichte des 19. Jahrhunderts - an der emphatisch als Hauptstadt des Jahrhunderts aufgefassten Stadt Paris anschaulich aus der Beschreibung der materialen Kultur der Zeit hervortreten zu lassen. Auch in diesem Zusammenhang ist die Figur des Momenthaften und der Unterbrechung entscheidend für Benjamins Bildbegriff: Die „Beziehung des Gewesnen [sic!] zum Jetzt“ wird als „dialektisch“ und „sprunghaft“ aufgefasst, nicht als Kontinuum (ebd., 577). Im Bild sucht Benjamin jenen Bruch des geschichtlichen Kontinuums zu verorten, der nicht Bild bleiben kann, sondern zur Sprache kommen muss. Tatsächlich ist sein Bilder-Denken auf die Sprache als dem eigentlichen Milieu und Medium des dialektischen Bildes gerichtet und liefert das kritische Instrument der Darstellung und der Übersetzung; durch sie lässt sich die Unterbrechung und Störung des blitzhaft aufscheinenden Bildes auf den „Ursprung der Gestaltungen und Veränderungen“ (ebd.) übertragen. Die Sprache ist schließlich für Benjamin das Scharnier, das ihm erlaubt, die Begriffe des Bildes, der Allegorie, des Denkbildes und des dialektischen Bildes ineinander übergehen zu lassen und als Schauplätze einer sich blitzartig herstellenden Einsicht in Anspruch zu nehmen.

In den 1930er Jahren widmete sich Benjamin den optischen Bildmedien Fotografie und Film. In den Aufsätzen „Kleine Geschichte der Photographie“ (1931) und „Das Kunstwerk im Zeitalter seiner technischen Reproduzierbarkeit“ (1935) nahm er die technisch-mediale Erscheinung des Kunstwerks in den Blick. Eine prominente Stellung in der Benjamin-Rezeption hat vor allem die medientheoretische Reflexion des Zusammenhangs von technischer Reproduzierbarkeit und Auraverlust des Kunstwerks eingenommen. Bedingt durch die massenhafte Reproduktionsmöglichkeit der Kunst, sieht er eine kollektive Veränderung der Kunstwahrnehmung sich vollziehen, die zum Auraverlust des Kunstwerks führt. So kontrovers diskutiert diese These heute auch ist, sie hat doch die Entwicklung einer allgemeinen Theorie der Medien in der Moderne nachhaltig geprägt. Für Benjamin war die Aura das, was nur noch in Verfallsformen sich zeigte. Im „Kunstwerk“-Aufsatz definiert er sie als „sonderbares Gespinst aus Raum und Zeit“, als „einmalige Erscheinung einer Ferne, so nah sie sein mag“(Benjamin 1980a [1935], 440). Daraus erklärt sich seine Faszination für die Paris-Fotografien des Eugène Atget (Abb. 2). Wie im hier abgebildeten Foto einer stillen Straße 
neben dem Panthéon zu erkennen, ist das Bild durch eine Leere, durch das NichtErreichbare geprägt. Solche Figuren der Unverfügbarkeit im Bild liest Benjamin im Kontext seiner Theorie der ,Ent-Auratisierung، des Bildes. Atgets neue FotoÄsthetik leite „die Befreiung des Objekts von der Aura ein“ und habe das Potential, auch der Literatur neue Impulse zu verleihen. Benjamin erhoffte sich nicht weniger als „literarische Pointierungen von Motiven, die Atget entdeckte“ (ebd., 378).

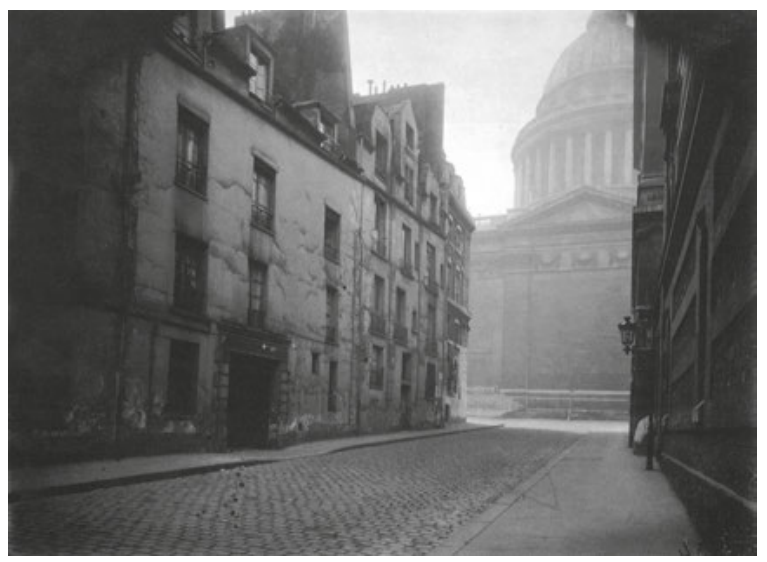

Abb. 2: Eugène Atget: Coin de la rue Vallette/Pantheon, Mars 1925, Fotografie, 1925

Der Umstand, dass Benjamin den ursprünglich theologisch gefärbten Aurabegriff für die säkulare Erfahrung der Moderne in Anwendung bringt, ist bezeichnend für die epistemische Struktur einer Reihe von ähnlich gelagerten Begriffsbildungen. Benjamins Kritik am Symbolbegriff gründete vor allem in seiner Kritik an einer repräsentationistisch verstandenen Auffassung des Symbols und verband sich mit seiner Hinwendung zum Begriff der Allegorie. Diese Chiffre wurde später für viele Autoren und Autorinnen im Umfeld der Dekonstruktion und des Poststrukturalismus wegweisend. In anderen Kontexten aber - zum Beispiel in seinem Buch Berliner Kindheit um Neunzehnhundert (1932-1938), im PassagenWerk (1928-1940) und in den Studien zu Proust (1929) - gelangte Benjamin zu einer positiveren Einschätzung des Symbols und im Zuge dessen zu einer Neubewertung der Rolle des Bildes in der Sprache. Benjamin entwickelt in diesen Schriften ein Symbolkonzept jenseits der Logik der reinen Repräsentation. So etwa in der Berliner Kindheit, wo er Transformationsprozessen zwischen an sich unähnlichen, nämlich grafischen, phonetischen und semantischen Ebenen im Rahmen einer allgemeinen Semiotik nachgeht. Diese Semiotik ist nicht auf das sprachliche Zeichen begrenzt, sondern versucht, „die Welt als Ganzes zeichenhaft zu lesen“ und betrifft insofern auch den Begriff des Symbols (Lemke 2008 
58). Entscheidend ist in diesem Zusammenhang Benjamins Rekonfiguration des Begriffs der Ähnlichkeit. Da alle Dinge - insofern sie Zeichen sind - aufeinander verweisen können, tragen sie zwar Spuren eines ursprünglichen mimetischen Verhaltens des Menschen, lösen diese Spuren der Ähnlichkeit im historischen Prozess aber gewissermaßen wieder auf. Daher stünden Zeichen und insbesondere Sprache und Schrift grundsätzlich im Modus einer „unsinnlichen“ oder auch „entstellten Ähnlichkeit“; sie seien stets Ergebnis von Übersetzungsprozessen (Benjamin 1980c [1933], 212; vgl. Waldow 2006, 98-99; Weigel 1997, passim).

Für Benjamin wird der kategoriale Gegensatz von Bild und Schrift hinfällig; in den Vordergrund tritt ihre gegenseitige Übersetzbarkeit. Die in der Berliner Kindheit geschilderte Erinnerung ist als ein Symbolisierungsprozess zu verstehen, der sich aus der Übersetzung zwischen Bild, Sprache und Sprachbild ergibt: „Die in der Kindheitserinnerung aufgerufenen Bilder sind weniger Verweise auf natürliche Referenten, sondern folgen als Sprachbilder den Gesetzen der Verknüpfung ihrer Zeichen als Entstehen, Gleiten und Verschwinden“ (Lemke 2008, 83). Benjamin hebt den Aspekt der entstellten Ähnlichkeit nicht nur an der Ding- und Zeichenwelt hervor, sondern ebenso an den durch technische Medien erzeugten Bildern und Tönen, an Fotografie, Fonografie, Kinematografie. Entsprechend heißt es im Passagen-Werk: „Daß zwischen der Welt der modernen Technik und der archaischen Symbolwelt der Mythologie Korrespondenzen spielen, kann nur der gedankenlose Betrachter leugnen.“ (Benjamin 1982 [1928-1940], 576).

An dieser Stelle lässt Benjamins Zeichentheorie deutliche Parallelen $\mathrm{zu}$ anderen Theorien des Bilder-Denkens, wie etwa denjenigen Warburgs, Freuds oder Cassirers, erkennen. Bereits die Funktion der ,Entstellung' in Berliner Kindheit weist zahlreiche Beziehungen zum psychoanalytisch informierten Begriff des symbolischen Bildes auf: Wie in der Traumdeutung Freuds oder in Warburgs Mnemosyne-Atlas ist es die Dynamik der Verdichtung und Verschiebung, die die entstellte Ähnlichkeit der Erinnerungs- und Sprachbilder hervorbringt. Die Entstellung avanciert für Benjamin zu einem Charakteristikum der Semiose und Medialität im Allgemeinen. Mit Cassirers Symboltheorie (die er nicht rezipierte) verband ihn die Auffassung, dass der Prozess der Symbolisierung einhergehe mit zunehmender Entstellung der „mythischen Namenssprache“ oder auch reinen Sprache, die nur noch als Leerstelle oder als mythische Erinnerung aufscheint (vgl. Waldow 2006, 266-267). Anders als Cassirer verknüpfte Benjamin, vor allem im Passagen-Werk, seine Reflexion des Entstellungsprozesses mit dem Studium der konkreten, historisch und gesellschaftlich imprägnierten Materialität der Zeichen.

Das variantenreiche Bilder-Denken Benjamins führt über ganz unterschiedliche Stationen: Allegorie und Allegorese, Symbol, Denkbild, dialektisches Bild, Vexier- oder Kippbild, Erinnerungsbild und Traumbild (vgl. Baumann 2002). In 
allen Bilddiskussionen ist aber nicht das Bild allein Ziel der Auseinandersetzung, sondern die Geburt des Bildes aus der Sprache, die Deutung des Bildes als Sprache und die Übersetzbarkeit des Bildes in andere Sprachen und Medien. Schon der Umstand, dass Benjamin den bildtheoretischen Reichtum seiner Überlegungen zunächst aus der Kritik der Literatur schöpfte und auch seine späteren kulturellen und historischen Analysen in direkter Verbindung mit der Literatur entwickelte - man denke nur an die zentrale Stellung Baudelaires, des Surrealismus und Prousts in seinem Werk -, macht den Beitrag dieses Denkens zu einer ,Bildwissenschaft der Literatur` deutlich. Die Figuren und Sprach-Bilder der Ähnlichkeit, der Mimesis und der Entstellung, des Erinnerns und des Vergessens, der Verschiebung und der Verdichtung, der Spur und des Entzugs stehen zwar in dem großen philosophischen Rahmen der Auseinandersetzung mit dem Mythos, der Theologie und der Säkularisierung. Ihr primärer Ort aber ist für Benjamin die Reflexion und Kritik der Sprache, und ihr Ziel eine Beschreibung der poiesis und des Lesens: „Jede Gegenwart ist durch diejenigen Bilder bestimmt, die mir ihr synchronistisch sind: jedes Jetzt ist das Jetzt einer bestimmten Erkennbarkeit. [...] Das gelesene Bild [...] trägt den Stempel des kritischen, gefährlichen Moments, welcher allem Lesen zugrunde liegt.“ (Benjamin 1982 [1928-1940], 578).

Das Bilder-Denken Warburgs, Cassirers und Benjamins entfaltet sich im Zuge einer epistemischen Wende in den 1920er Jahren, die, äußerst folgenreich, der veränderten kulturellen Funktion der Visualität Rechnung trägt (siehe auch 2.8 STIEGLER). Dass das Bild in der medialen Abgrenzung von Sprache und Literatur reflektiert werden müsse und zugleich durch seine Übersetzung in literarischsprachliche Prozesse Kontur gewinnt - in dieser Orientierung kommen die drei Autoren trotz unterschiedlicher Akzentuierung ihrer Bildkonzepte überein. Begriffe wie Geste, Ausdruck und Darstellung rücken in den Vordergrund des kulturwissenschaftlich informierten Bilder-Denkens. Benjamin und Warburg werten es durch Aspekte wie Diskontinuität, Unterbrechung, Krise und Symptom auf. Anders Cassirer: Sein Symbolbegriff formuliert ein kulturelles Konzept der Imagination, für die das Bild ein unverzichtbares Zwischenstadium auf dem Weg zur abstrakten Darstellung ist. Es ist der Symbolbegriff und es sind nicht die expliziten Aussagen zum Bild, in dem sich eine Korrespondenz zu den Bildkonzepten Warburgs und Benjamins zeigt.

\section{Literaturverzeichnis}

Baumann, Valérie. Bildnisverbot. Zu Walter Benjamins Praxis der Darstellung: Dialektisches Bild, Traumbild, Vexierbild. Eggingen: Edition Isele, 2002. 
Benjamin, Walter. „Das Kunstwerk im Zeitalter seiner technischen Reproduzierbarkeit. Erste Fassung“ [1935]. Gesammelte Schriften Bd. 1.2: Abhandlungen. Hrsg. von Rolf Tiedemann und Hermann Schweppenhäuser. Frankfurt am Main: Suhrkamp, 1980a. 431-469.

Benjamin, Walter. „Goethes Wahlverwandtschaften“ [1924]. Gesammelte Schriften Bd.1.1: Abhandlungen. Hrsg. von Rolf Tiedemann und Hermann Schweppenhäuser. Frankfurt am Main: Suhrkamp, 1980b. 123-201.

Benjamin, Walter. „Über das mimetische Vermögen“ [1933]. Schriften Bd. 2.1: Aufsätze, Essays, Vorträge. Hrsg. von Rolf Tiedemann und Hermann Schweppenhäuser. Frankfurt am Main: Suhrkamp, 1980c. 210-213.

Benjamin, Walter. „Über den Begriff der Geschichte“ [1942]. Gesammelte Schriften Bd.1.2: Abhandlungen. Hrsg. von Rolf Tiedemann und Hermann Schweppenhäuser. Frankfurt am Main: Suhrkamp, 1980d. 691-706.

Benjamin, Walter. „Ursprung des deutschen Trauerspiels“ [1925]. Gesammelte Schriften Bd. 1.1: Abhandlungen. Hrsg. von Rolf Tiedemann und Hermann Schweppenhäuser. Frankfurt am Main: Suhrkamp, 1980e. 202-430.

Benjamin, Walter. „Zentralpark“ [1938]. Gesammelte Schriften Bd. 1.2: Abhandlungen. Hrsg. von Rolf Tiedemann und Hermann Schweppenhäuser. Frankfurt am Main: Suhrkamp, 1980 f. 657-690.

Benjamin, Walter. Gesammelte Schriften Bd. 5.1: Das Passagen-Werk. Hrsg. von Rolf Tiedemann. Frankfurt am Main: Suhrkamp, 1982 [1928-1940].

Benjamin, Walter. Gesammelte Briefe. Bd. 1: 1910-1918. Hrsg. von Gershom Scholem und Theodor W. Adorno. 2. Aufl. Frankfurt am Main: Suhrkamp, 1993.

Böhme, Hartmut. „Aby M. Warburg“. Klassiker der Religionswissenschaft. Hrsg. von Axel Michaels. München: Beck. 1997. 133-156.

Cassirer, Ernst. „Der Begriff der symbolischen Form im Aufbau der Geisteswissenschaften“ [1921-1922]. Wesen und Wirkung des Symbolbegriffs. 7. Aufl. Darmstadt: Wissenschaftliche Buchgesellschaft, 1983. 169-200.

Cassirer, Ernst. Philosophie der symbolischen Formen. Bd. 1. 9. Aufl. Darmstadt: Wissenschaftliche Buchgesellschaft, 1988 [1923].

Cassirer, Ernst. Philosophie der symbolischen Formen. Bd. 2. 8. Aufl. Darmstadt: Wissenschaftliche Buchgesellschaft, 1987 [1925].

Cassirer, Ernst. Philosophie der symbolischen Formen. Bd.3. 8. Aufl. Darmstadt: Wissenschaftliche Buchgesellschaft, 1982 [1929].

Cassirer, Ernst. Versuch über den Menschen. Einführung in eine Philosophie der Kultur. Übers. von Reinhard Kaiser. Frankfurt am Main: Fischer, 1990 [1944].

Cassirer, Ernst. „Der Begriff der symbolischen Form im Aufbau der Geisteswissenschaften“ [1922]. Aufsätze und kleine Schriften (1922-1926). Hrsg. von Birgit Recki. Hamburg: Meiner, 2003a. 75-104.

Cassirer, Ernst. Goethe-Vorlesungen (1940-1941). Ernst-Cassirer-Nachlassausgabe Bd.11. Hrsg. von John M. Krois Hamburg: Meiner, 2003b.

Cassirer, Ernst. Kleinere Schriften zur Goethe und zur Geistesgeschichte. Ernst-CassirerNachlassausgabe. Bd. 10. Hrsg. von Barbara Naumann. Hamburg: Meiner, 2006 [1925-1944].

Didi-Huberman, Georges. „Dialektik des Monstrums. Aby Warburg und das Paradigma des Symptomalen“. Bilder-Denken. Bildlichkeit und Argumentation. Hrsg. von Barbara Naumann und Edgar Pankow. München: Fink, 2004. 203-233. 
Didi-Huberman, Georges. „Das Nachleben der Bilder“. Kunstgeschichte und Phantomzeit nach Aby Warburg. Übers. von Michael Bischoff. Frankfurt am Main: Suhrkamp, 2010 [2002].

Hansen, Miriam Bratu. „Benjamin's Aura“. Critical Inquiry 34 (2008): 336-375.

Hensel, Thomas. Wie aus der Kunstgeschichte eine Bildwissenschaft wurde. Aby Warburgs Graphien. Berlin: Akademie, 2011.

Krois, John Michael. „Die Universalität der Pathosformeln“. Körperbilder und Bildschemata. Hrsg. von Horst Bredekamp und Marion Lauschke. Berlin: Akademie, 2011. 76-91.

Lemke, Anja. Gedächtnisräume des Selbst. Walter Benjamins „Berliner Kindheit um neunzehnhundert. 2. Aufl. Würzburg: Königshausen und Neumann, 2008.

Mitchell, William J. T. „The Pictorial Turn“. Artforum 30 (1992): 89-94.

Naumann, Barbara. Philosophie und Poetik des Symbols. Cassirer und Goethe. München: Fink, 1998.

Naumann, Barbara, und Edgar Pankow (Hrsg.). Bilder-Denken. Bildlichkeit und Argumentation. München: Fink, 2004.

Recki, Birgit. Kultur als Praxis. Eine Einführung in Ernst Cassirers Philosophie der symbolischen Formen. Berlin: Akademie, 2004.

Waldow, Stephanie. Der Mythos der reinen Sprache: Walter Benjamin, Ernst Cassirer, Hans Blumenberg. Paderborn: Fink, 2006.

Walzel, Oskar. Die wechselseitige Erhellung der Künste. Ein Beitrag zur Würdigung kunstgeschichtlicher Begriffe. Berlin: Reuther \& Reinhard, 1917.

Warburg, Aby. Das Schlangenritual. Ein Reisebericht. Mit einem Nachwort von Ulrich Raulff. Berlin: Wagenbach, 1988 [1923].

Warburg, Aby. Ausgewählte Schriften und Würdigungen. Hrsg. von Dieter Wuttke. 3. Aufl. Baden-Baden: Valentin Körner, 1992.

Warburg, Aby. „Italienische Kunst und internationale Astrologie im Palazzo Schifanoja zu Ferrara“ [1912]. Ausgewählte Schriften und Würdigungen. Hrsg. von Dieter Wuttke. 3. Aufl. Baden-Baden: Valentin Körner, 1992.173-198.

Warburg, Aby. Die Erneuerung der heidnischen Antike. Kulturwissenschaftliche Beiträge zur Geschichte der europäischen Renaissance. Gesammelte Schriften Bd.1. Repr. d. Ausg. von Gertrud Bing u. Mitarb. von Fritz Rougemont [1932]. Neu hrsg. von Horst Bredekamp, und Michael Diers et al. Berlin: Akademie, 1998.

Warburg, Aby. Der Bilderatlas Mnemosyne. Hrsg. von Martin Warnke unter Mitarbeit von Claudia Brink. 4. Aufl. Berlin: Akademie, 2012a [ca. 1927].

Warburg, Aby. „Italienische Antike im Zeitalter Rembrandts“ [1926]. Nachhall der Antike. Aby Warburg. Zwei Untersuchungen. Hrsg. von Pablo Schneider. Zürich: Diaphanes, 2012b.

Weigel, Sigrid. Entstellte Ähnlichkeit. Walter Benjamins theoretische Schreibweise. Frankfurt am Main: Suhrkamp, 1997.

Weigel, Sigrid. Walter Benjamin. Die Kreatur, das Heilige, die Bilder. Frankfurt am Main: Fischer, 2008.

Zumbusch, Cornelia. Wissenschaft in Bildern. Symbol und dialektisches Bild in Aby Warburgs Mnemosyne-Atlas und Walter Benjamins Passagenwerk. Berlin: Akademie, 2004. 